\title{
Justice, chance et responsabilité dans la santé et les soins
}

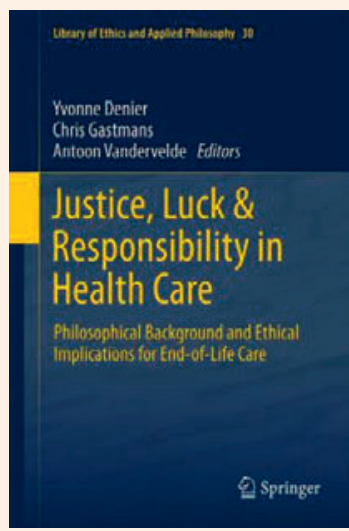

Yvonne Denier, Chris Gastmans, Anton Vandervelde (ed.)

Justice, Luck \& Responsibility in Health Care.

Dordrecht: Springer; 2012

242 p., $144.40 \mathrm{CHF}$

ISBN 978-9- 4007-5334-1

* Dont elle dit qu'il est déjà bien admis et établi. Mais ce n'est pas le cas en Suisse où, si certaines formes de diagnostic prénatal ont une dimension génétique (amniocentèse pour recherche de trisomie par exemple), le diagnostic préimplantatoire (DPI) reste interdit à ce stade. Mais en Belgique d'où est l'auteur, Bruxelles a un très important centre de DPI, auquel ont recours des couples de Suisse.

1 Denier Y, Gastmans C, Vandervelde A (ed.). Justice, Luck \& Responsibility in Health Care. Dordrecht: Springer; 2012.

2 Martin J. Directives anticipées et démence - des aspects indécidables? Bull Méd Suisses. 2013;94(8):312.
La notion de chance, aux plusieurs sens de «bénéfice» (avoir de la chance), de probabilité (combien de chances que..?) et de hasard, joue un rôle majeur dans les débats traitant de santé, d'assurance-maladie, de contrainte à cet égard (couverture universelle), de responsabilité des pouvoirs publics. Ceci aussi bien dans la réflexion et l'action des citoyens individuels (cela vaut-il la peine que je complète ma couverture par une police privée? Est-il vraiment utile que je me plie à un mode de vie sain?) que dans celles des gouvernements et parlements. L'exemple majeur actuel sont les Etats-Unis, du point de vue de l'abondance des controverses publiques comme dans la production de travaux académiques (notamment avec le passage difficile il y a deux ans de «Obamacare», la loi qui entend apporter des soins à un coût supportable à la grande majorité de la population).

Justice, Luck \& Responsibility in Health Care, publié sous la direction de trois philosophes de l'Université de Louvain/Leuven, restitue les exposés d'une conférence tenue en mai 2011 [1]. Ouvrage sophistiqué (au bon sens du terme); les 12 auteurs sont des enseignements universitaires en philosophie et éthique; trois d'entre eux avec aussi une formation théologique et trois autres qui incluent l'économie et la science politique dans leurs travaux. Sans qu'il s'agisse d'une lecture carrément distrayante, il n'y a en général pas de problème de compréhension de ce qui est exposé.

Amusant de noter qu'en exergue - bien trouvé apparaît la fable de la Fontaine «La cigale et la fourmi».

\section{Le «naturel» n'est plus comme auparavant un donné, on peut agir sur lui - ce qui implique des prises de responsabilité}

Yvonne Denier explore les questions de justice et responsabilité en rapport particulièrement avec le diagnostic génétique prénatal*. Elle cite Buchanan et al. (les citations sont ma traduction de l'anglais): «A mesure que les possibilités d'interventions génétiques sur des êtres humains deviennent plus proches d'être concrétisées, nous serons probablement forcés d'élargir radicalement notre conception de la justice, en incluant des attributs naturels aussi bien que sociaux parmi les biens [choses/chances] que des institutions justes doivent réguler/distribuer équitablement.» Ces auteurs parlent de la «colonisation du naturel par le juste».

Denier relève que, aujourd'hui, nos caractéristiques naturelles ne sont plus une pure question de chance (brute luck). «La frontière cruciale entre chance et choix/hasard est l'épine dorsale de notre éthique et de notre moralité et un quelconque glissement à cet égard est très disloquant, en nous demandant de repenser des postulats fondamentaux sur les relations entre justice, nature humaine et progrès moral»; entre autres le postulat que «les résultats de la loterie naturelle dépendent strictement de la chance et ainsi échappent à notre responsabilité morale».

Dans sa contribution, Vandervelde évoque une question très délicate, qu'on a envie de refuser mais dont il serait surprenant qu'elle ne soit pas posée plus souvent à l'avenir: «Aujourd'hui déjà le diagnostic génétique prénatal permet de prédire des déficiences chromosomiques de l'enfant à naître. Est-ce que la justice égalitaire demandera de maintenir une solidarité avec des parents qui, le sachant et le voulant, choisissent de donner naissance à un enfant handicapé?». On peut s'interroger de manière comparable sur les parents refusant de faire vacciner leurs enfants, ou ceux qui sciemment les soumettent à d'autres risques évitables (sur ce thème, voir «Yes, they should!» puis «Counterconsiderations», p. 6-12).

\section{Questions autour de la démence}

J'ai parlé récemment de ce sujet [2]. Thomas Nys, d'Amsterdam, développe une réflexion sur les places respectives de ce qu'il appelle (de même que den Hartogh) critical interests, à savoir ceux qui apparaissent dans les directives établies alors que le patient avait le discernement, et experiential interests, ceux que la personne démente manifeste sur le moment - dont il y a lieu de reconnaître que l'interprétation n'est pas aisée. Il discute le rôle des proches: «Nous avons généralement une bonne idée de qui sont les personnes importantes pour le patient (significant others). Les membres de la famille ont déjà voix au chapitre dans le traitement de leurs proches. Mon intention était de fournir quelques justifications théoriques de ce rôle et de prendre du recul par rapport au soupçon fréquent à leur égard - parce qu'ils seraient de médiocres interprètes des désirs du patient ou qu'ils seraient influencés par leur propres intérêts, et par conséquent qu'on ne saurait leur faire confiance.» A l'évidence, il n'y a pas ici de recette valable dans tous les cas et cela sollicite des appréciations fines des soignants. Ecoute, doigté, bon sens, sont requis.

Citation d'une expérience souvent vécue: «Mon mari [souffrant de démence] n'est plus l'homme que 
j'ai épousé. Ce dernier est mort depuis deux ans.» En deçà des grandes questions philosophiques, comment contester que cela corresponde à une réalité dans la vie de l'épouse?

\section{Rationnement, devoir de mourir(!)}

Les ressources sont toujours en quantité limitée mais la notion de rationnement reste largement frappée d'anathème dans nos pays - où le débat politique à ce sujet semble du registre de l'impossible [3].

Pourtant n'est il pas souvent vrai, comme le dit Martin Gunderson, que «si un gouvernement ne développe pas de politique de rationnement, la distribution de ressources rares et onéreuses en matière de soins sera réalisée par d'autres moyens, comme la couverture par des assurances privées ou la capacité propre de payer».

John Hardwig est un philosophe américain auteur de plusieurs articles engagés sur le «devoir de mourir» dans certaines situations (plus fréquentes aux EtatsUnis où il est courant d'être ruiné par le coût des soins). Par les progrès de la médecine, dit-il, nous sommes confrontés à «a new kind of death». A son avis, même s'ils sont substantiels (comme en Europe), «les filets de sécurité sociale institutionnels ne couvriront jamais toutes les difficultés que peuvent rencontrer les familles et les proches d'un malade. Pour cette raison, les dispositions [collectives] n'élimineront jamais la nécessité de faire face de manière responsable à la fin de sa propre vie» (dans l'esprit de l'auteur, d'admettre parfois le devoir de s'en aller). Ajoutant: «Si cela semble horrible, je peux seulement inciter chacun d'entre nous à se souvenir que le devoir de mourir n'est que l'autre face de la meilleure santé et des vies plus longues dont nous sommes privilégiés de bénéficier». Antoon Vandervelde, de Louvain: «Que requiert la justice à la fin de la vie? La médecine a permis de retarder la mort naturelle pour une très longue période, à un coût financier considérable pour la société et souvent la famille. Devenons-nous en tant qu'individus tenus de prendre la responsabilité du moment où nous mourrons?».

\section{Les maladies infectieuses, sujet classique de santé publique}

"Comme la transmission des maladies infectieuses peut être liée à la sphère privée des citoyens, les mesures prises par les autorités de santé publique vont souvent entrer en conflit avec la protection des libertés civiles» (Jeroen Luyten). Et plus loin: «Pour réduire l'incidence de ces maladies de la manière la moins controversée, les autorités devraient pouvoir compter largement sur la coopération des citoyens.» C'est certainement ce que le médecin de santé publique qui signe cette recension espère. Au reste, rappel utile, «le niveau souhaité/requis de prévention dépendra de la valeur accordée à la santé de la population par rapport à d'autres objectifs sociétaux qui devraient cas échéant être sacrifiés».

\section{Donner suffisamment attention au contexte sociétal}

Citation de l'épilogue du livre, par Paul Schotsmans, personnalité senior de la bioéthique belge: «Bien trop longtemps, les bioéthiciens ont négligé le devoir de situer des responsabilités et défis hautement personnalisés> dans le contexte des systèmes et politiques de santé, au plan national et international. Progressivement toutefois, ils ont commencé à regarder audelà de leur micro-contexte et à intégrer les enjeux sociétaux. Personne ne vit seul. Personne ne naît comme une page strictement blanche. Nous sommes tous des êtres humains situés (...) Si nous n'incluons pas les débats dans le contexte sociétal, même s'agissant de choix qui semblent purement privés, nous agissons comme si nous étions des êtres surnaturels.»

Ce qui rappelle cette forte formule d'un auteur anonyme: «Les scientifiques et médecins ont fait des choses merveilleuses en regardant dans le microscope, il importe qu'ils regardent aussi par la fenêtre.» On peut parfois exprimer le même souhait pour ceux qui se préoccupent de bioéthique (dont je suis!).

Trop souvent, on voit la confrontation de convictions fortement ancrées, pas vérifiées dans la réalité autour de nous mais auxquelles on tient mordicus. «Ne m'apportez pas des faits, j'ai déjà mes idées!», dit à cet égard une amie dirigeant une organisation d'aide sociale.

\section{C'est de vie humaine qu'il s'agit}

En résumé, un ouvrage d'importance actuelle tant les questions de justice, de dignité, d'autonomie de la personne et de devoir de s'assumer, du rôle nécessaire (et suffisant?) des pouvoirs publics, vont continuer à être très présentes et discutées. Discutées et disputées, dans la mesure où chacune de ces notions reçoit des définitions diverses selon le mode de raisonnement et les fondements philosophiques et sociaux des experts ou des politiques qui s'expriment - et qu'il est illusoire d'espérer par l'argumentation amener à une même position...

Ainsi, qu'appelons-nous une vie vraiment humaine. Herman De Djin: «Qu'est-ce qui lui donne sa valeur? Est-ce qu'elle consiste à avoir eu un certain nombre d'années de vie d'un certaine qualité? Est-ce qu'une bonne vie est d'avoir vécu des expériences de diverses natures? Ou une vie véritablement humaine doit-elle être jugée de manière holistique, selon la réalisation de fins ou buts supérieurs? Dans ce cas, cela signifie-t-il que la valeur d'une vie ne peut être déterminée que dans le contexte d'une reconnaissance par d'autres? Qu' entendons-nous exactement en disant que les humains sont des êtres relationnels? [Mitsein]» (voir aussi p. 18-20). Bonnes questions.

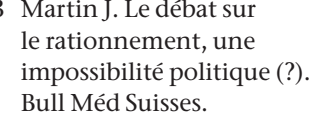

